\title{
Predictive Value of Antiviral Effects in the Development of Hepatocellular Carcinoma in the General Korean Population with Chronic Hepatitis B
}

\author{
Jae-Jun Shim ${ }^{1}$, In Hwan $\mathrm{Oh}^{2}$, Sang Bae Kim ${ }^{3}$, Jung Wook Kim ${ }^{1}$, Chang Kyun Lee ${ }^{1}$, Jae Young Jang ${ }^{1}$, Ju-Seog Lee ${ }^{4}$, and \\ Byung-Ho Kim ${ }^{1}$ \\ Departments of ${ }^{1}$ Internal Medicine and ${ }^{2}$ Preventive Medicine, Kyung Hee University School of Medicine, Seoul, Korea, ${ }^{3}$ Human Genome \\ Sequencing Center, Baylor College of Medicine, and ${ }^{4}$ Department of Systems Biology, University of Texas MD Anderson Cancer Center, \\ Houston, TX, USA
}

Background/Aims: The benefit of oral antiviral therapy in preventing hepatocellular carcinoma (HCC) in the general population is not well understood. We used a novel prediction method to estimate the risk of HCC in the Korean population based on various treatment guidelines. Methods: The 5-year risk of HCC following antiviral therapy was calculated using an HCC risk prediction model. A virtual cohort that represented Koreans ( $>40$ years old) with chronic hepatitis $B$ virus (HBV) infection was established using the fifth National Health and Nutrition Examination Survey. The antiviral indications tested were the Korean National Health Insurance (NHI) and European Association for the Study of the Liver (EASL) guidelines as well as a new extended indication (serum HBV DNA $>2,000 \mathrm{IU} / \mathrm{mL}$ regardless of serum aminotransferase level). Results: A total of 993,872 subjects were infected with HBV in the general Korean population. Over a 5-year period, 2,725 HCC cases were predicted per 100,000 persons $(0.55 \% / y r)$. When the cohort was treated based on the Korean NHI, the EASL, and the newly extended indications, HCC risks decreased to 2,531 (-7.1\%), 2,089 (-23.3\%), and $1,122(-58.8 \%)$ cases per 100,000 persons, respectively $(\mathrm{p}<0.0001)$. Conclusions: Simulated risk prediction suggests that extending of oral antiviral indication may reduce the HCC risk in the general population. (Gut Liver 2016;10:962-968)

Key Words: Antiviral therapy; Hepatitis B, chronic; Carcinoma, hepatocellular

\section{INTRODUCTION}

Chronic hepatitis B virus (HBV) infection is the primary cause of hepatocellular carcinoma (HCC), accounting for approximately 75\% of its underlying etiologies in Korea. ${ }^{1}$ Although the national vaccination program markedly reduced HBV infection in the younger population ( $<30$ years), the rate of HBV infection in middle-aged and elderly individuals is high. ${ }^{2}$ According to a national survey, approximately $4 \%$ of Korean adults over the age of 40 are affected by chronic HBV infection. ${ }^{2}$ Since 95\% of HCC cases occur in this age group, more aggressive strategies are required to prevent HCC in Korea. ${ }^{3}$

Over the last two decades, knowledge of the relationship between chronic HBV infection and HCC development has increased. Chronic HBV infection-including inactive HBV carrier status-is associated with development of $\mathrm{HCC}^{4-6}$ A higher level of HBV replication, older age, male gender, and higher serum alanine aminotransferase (ALT) levels are independent predictors of the development of HCC. ${ }^{4}$ Using these parameters, various prediction models of HCC risk have been developed and validated. ${ }^{7,8}$ The individual risk of developing HCC can be predicted if the patient was untreated. Recently, antiviral treatment with oral nucleos(t)ide analogues has shown promising results in patients with chronic hepatitis B (CHB). Several clinical trials and observational studies have reported beneficial effects of antiviral treatment in terms of HCC prevention through persistent viral suppression. ${ }^{9-11}$ Although the preventive effect is imperfect, such viral suppression can reduce substantially the risk of HCC development in patients with chronic HBV infection.

The possibility of predicting the risk of HCC development even in patients receiving antiviral treatment remains unclear.

Correspondence to: Byung-Ho Kim

Department of Internal Medicine, Kyung Hee University Hospital, Kyung Hee University School of Medicine, 23 Kyungheedae-ro, Dongdaemun-gu, Seoul 02447, Korea

Tel: +82-2-958-8145, Fax: +82-2-968-1848, E-mail: kimbh1955@gmail.com

Received on August 27, 2015. Revised on December 30, 2015. Accepted on January 5, 2016. Published online June 13, 2016 pISSN 1976-2283 eISSN 2005-1212 https://doi.org/10.5009/gnl15426

@ This is an Open Access article distributed under the terms of the Creative Commons Attribution Non-Commercial License (http://creativecommons.org/licenses/by-nc/4.0) which permits unrestricted non-commercial use, distribution, and reproduction in any medium, provided the original work is properly cited. 
Because an antiviral treatment could suppress significantly HBV replication and reduce necroinflammation and fibrosis in the liver, such improvements might affect the parameters used in the HCC prediction models. Interestingly, after treatment with entecavir, a potent antiviral agent, the prediction models predicted the risk of HCC more accurately than did baseline models. ${ }^{12}$ Based on this finding, we assumed that accurate prediction of the risk of HCC before and during antiviral treatment in patients with chronic HBV infection is possible.

Since the introduction of oral nucleot(s)ide analogues, the Korean National Health Insurance $(\mathrm{NHI})^{13}$ has updated inclusion criteria for antiviral therapy against CHB based on serum aminotransferase and HBV DNA levels. Even though the inclusion criteria have been gradually extended, many patients are unable to start antiviral therapy until they meet the stringent criteria. Since the NHI indications focus on higher-risk patients, the majority of CHB patients are excluded from treatment. However, the number of patients that can be treated using the $\mathrm{NHI}$ indication criteria remains unknown and there are insufficient data on the current status of chronic HBV infection in the Korean population. To make antiviral therapy cost-effective, an accurate estimate of at-risk patients and prediction of treatment outcomes are required. Further, more patients should receive antiviral therapy to increase its beneficial effects. However, supporting data to extend antiviral indications are scarce.

To overcome these limitations, we used a novel computerbased modelling approach to predict HCC risk in the general population with HBV. We attempted to calculate the number of patients that could receive antiviral therapy and whether the current antiviral indications are suitable to reduce the HCC risk for the general Korean population.

\section{MATERIALS AND METHODS}

\section{Development of a representative cohort of chronic HBV infection}

We established a representative cohort using data from the fifth Korean National Health and Nutrition Examination Survey (KNHANES), ${ }^{14}$ a cross-sectional study conducted from 2010 to 2012. The KNHANES included all civilian noninstitutionalized Korean individuals who were selected to represent the Korean population according to geographical and demographic characteristics. The target population of the present study was hepatitis B surface antigen (HBsAg)-positive subjects over the age of 40 years, as the immune-tolerant phases are uncommon and the risk of HCC is increasing rapidly in these patients. Among 8,435 eligible subjects, 453 were identified as HBsAg-positive; 21 subjects who reported they had liver cirrhosis and 12 subjects who had missing data were excluded. Liver cirrhosis was clinically diagnosed when image findings (nodular liver surface) and laboratory findings (albumin, bilirubin, prothrombin time, or platelet counts) were consistent with cirrhosis. ${ }^{15}$ Consequently, the study enrolled 420 subjects (Fig. 1). We assumed that these subjects were representative of all noncirrhotic patients with HBV infection in Korea. Consequently, we estimated that 4.13\% (993,872 subjects) of 24,064,693 Korean individuals over the age of 40 years were HBsAg positive in 2011. As HBV DNA levels and hepatitis B envelope antigen (HBeAg) status were

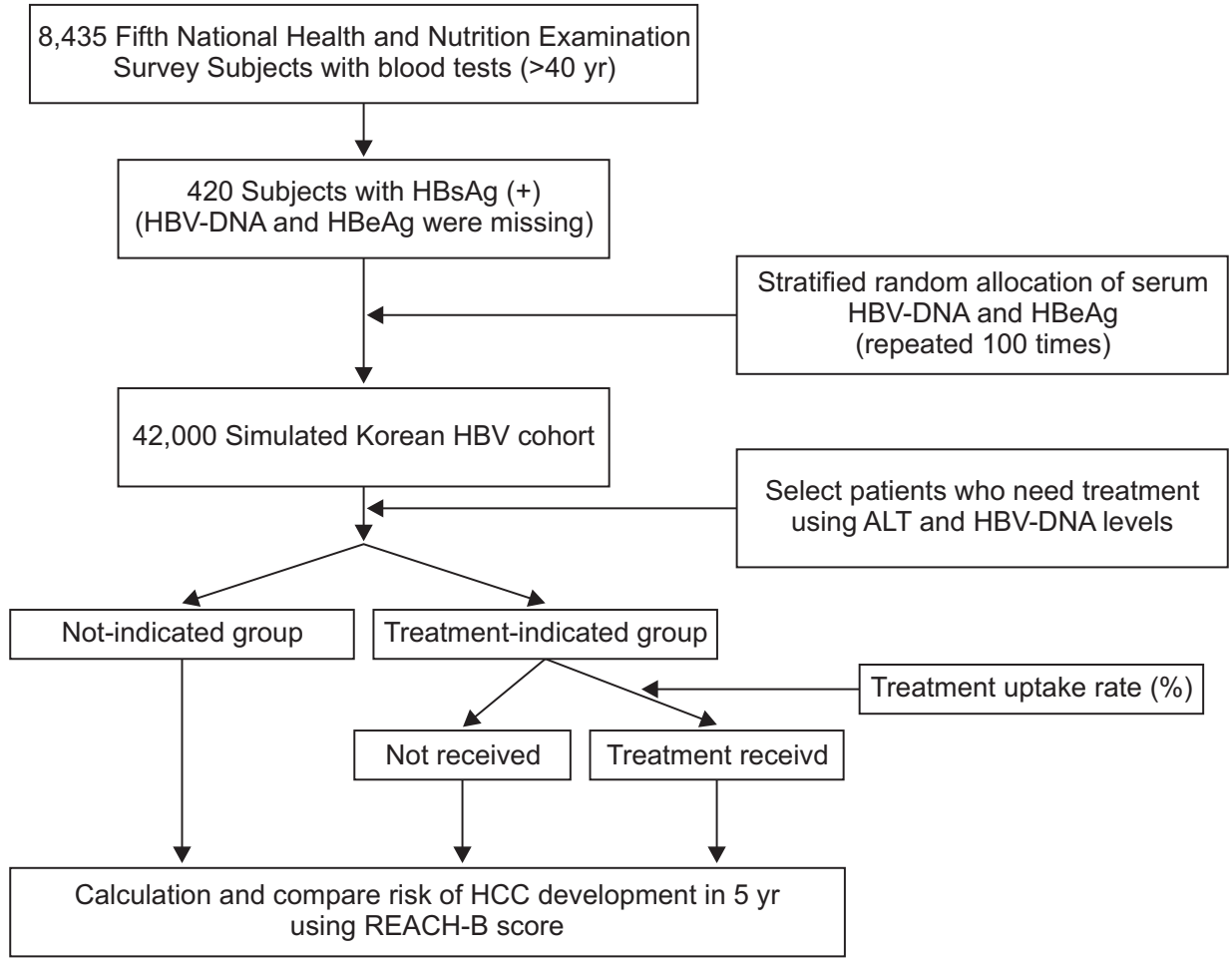

Fig. 1. Study flow chart. We constructed a Korean hepatitis B virus (HBV) cohort from the fifth National Health and Nutrition Examination Survey. Because serum HBV DNA levels and hepatitis B e antigen (HBeAg) positivity data were missing, HBV status was allocated randomly according to alanine aminotransferase (ALT) level. The distribution of HBV DNA and HBeAg was simulated using reported data from the Risk Evaluation of Viral Load Elevation and Associated Liver Disease/Cancer study. Random allocation was repeated 100 times. We compared three treatment indication guidelines for antiviral therapy. The risk of hepatocellular carcinoma (HCC) development in 5 years was calculated using Risk Estimation for HCC in Chronic Hepatitis B (REACHB) scores. 
Table 1. Indications for Antiviral Treatment Used in This Study

\begin{tabular}{lccc} 
& Korean NHI & EASL & New extended indication \\
\hline Liver enzyme & AST or ALT $\geq 80$ IU $/ \mathrm{L}$ & ALT $\geq \mathrm{ULN}^{*} \times 1$ \\
HBV DNA level, IU/mL & $\geq 20,000^{\dagger}$ & $\geq 2,000$ \\
\hline
\end{tabular}

NHI, National Health Insurance; EASL, European Association for the Study of the Liver; AST, aspartate aminotransferase; ALT, alanine aminotransferase; ULN, upper limit of normal; HBV, hepatitis B virus.

${ }^{*} \mathrm{ULN}$ was defined as $33 \mathrm{IU} / \mathrm{L}$ in men and $25 \mathrm{IU} / \mathrm{L}$ in women $;{ }^{17} \geq 2,000 \mathrm{IU} / \mathrm{mL}$ in case of hepatitis B e antigen (-) chronic hepatitis B or liver cirrhosis.

not available in the KNHANES data, we determined the status of HBV infection in Koreans based on Taiwanese data. Koreans and Taiwanese have similar modes of HBV transmission, mostly neonatal infection. HBV infection status was determined using the Risk Evaluation of Viral Load Elevation and Associated Liver Disease/Cancer-Hepatitis B Virus (REVEAL-HBV) study, a Taiwanese prospective community-based cohort study. Details of the study have been reported. ${ }^{4,7}$ Briefly, 4,155 participants who were seropositive for HBsAg and were HCC-free at the beginning of the study were enrolled. Among these, blood test data for 3,851 were available, and the distribution of HBV DNA levels and proportion of HBeAg positivity were evaluated. We extrapolated from the REVEAL study and randomly allocated HBV DNA levels (<60, 60-2,000, 2,000-20,000, 20,000-200,000, and $>200,000 \mathrm{IU} / \mathrm{mL})$ according to ALT levels $(<15,15-45,>45 \mathrm{U} /$ L). HBeAg positivity was also assigned according to the HBV DNA level. Random allocation was repeated 100 times and a large virtual cohort $(n=42,000)$ was made in one session. We constructed 20 virtual cohorts by repeating this process. Random allocation and statistical analyses were performed in an $\mathrm{R}$ language environment version 3.1.2 (http://www.r-project.org).

\section{Current and new extended treatment criteria in patients with HBV}

Diverse treatment guidelines are presently used in different countries based on their medical resources and the societal burden of chronic HBV infection. In South Korea, individuals are obligated to participate in the NHI. The NHI reimburses patients undergoing antiviral treatment if their laboratory results meet the following criteria: serum aspartate aminotransferase (AST) or ALT levels must be $>80 \mathrm{IU} / \mathrm{L}$ and the baseline serum HBV DNA levels must be $>2,000$ and $>20,000 \mathrm{IU} / \mathrm{mL}$ in $\mathrm{HBeAg}$ negative and positive $\mathrm{CHB}$ patients, respectively. If patients start antiviral treatment without meeting these criteria, obtaining reimbursement from the NHI is difficult, as HBV DNA and ALT levels could normalize during treatment. Therefore, most patients who do not meet the reimbursement criteria have to defer antiviral treatment until they satisfy the criteria. Consequently, clinical practice is markedly affected by economic status, rather than scientific evidence. We attempted to establish new treatment indications for patients with $\mathrm{CHB}$, designed to enroll all patients except for inactive HBV carriers (HBV DNA <2,000 $\mathrm{IU} / \mathrm{mL}$ ). We also used the recent European Association for the
Study of the Liver (EASL) guidelines,${ }^{16}$ as they include the widest treatment indication range. The treatment indications of the three guidelines used in this study are summarized in Table $1{ }^{17}$

\section{Estimation of patients who required antiviral treatment and prediction of treatment responses}

We determined the proportion of patients who were candidates for antiviral treatment according to the treatment criteria. To adjust for uncertainty in individuals who would receive treatment, the patients were selected randomly based on the treatment uptake rate, which was defined as the rate of participation in the treatment group among the indicated patients. For example, a treatment uptake rate of $60 \%$ meant that 60 of 100 patients were treated. As patient selection among the indicated could be very diverse, we used a random selection processes in an $\mathrm{R}$ language environment. We performed the selection process 100 times and the resulting average values were used. All patients were assumed to receive potent antiviral agents such as tenofovir or entecavir. We estimated the improvements in laboratory parameters after antiviral treatment based on their actual data or recent clinical trials. ${ }^{18-20}$ After 2 years of treatment, serum HBV DNA levels decreased to $<2,000 \mathrm{IU} / \mathrm{mL}$ in 95\% of treated patients, and serum ALT levels were normalized to the same distribution as seen in non-HBV-infected individuals. Moreover, the rate of HBeAg loss was 30\% after 2 years. ${ }^{21,22}$

\section{Prediction of risk for HCC development in the general Korean population}

The HCC risk was predicted using the risk estimation for HCC in chronic hepatitis B (REACH-B) score. ${ }^{8}$ The REACH-B score was developed based on the REVEAL-HBV study cohort consisting of 3,584 patients without cirrhosis. Predictive parameters included gender, age, serum ALT level, HBeAg status, and serum HBV DNA levels. The score comprised 17 points with HCC risk ranging from $0.0 \%$ to $23.6 \%$ at 3 years, $0.09 \%$ to $47.4 \%$ at 5 years, and $0.0 \%$ to $81.6 \%$ at 10 years. The score was validated in other cohorts from three hospitals in Hong Kong and South Korea and was demonstrated to estimate the risk of developing HCC at 3, 5, and 10 years accurately. It also predicted HCC risk within an acceptable range, especially in Asian patients on antiviral therapy. ${ }^{12}$

We estimated the risk of developing HCC at the 5-year point. We calculated the 3-year risk of HCC following 2 years of an- 
tiviral treatment in patients who received antiviral therapy. We predicted improvement in laboratory parameters after 2 years of treatment and used them for risk prediction. In patients who did not receive antiviral treatment, we used the 5-year risk of HCC using baseline variables. Then, we calculated the number of new HCC cases in the general Korean population, multiplying atrisk subjects by the estimated 5-year risk (\%). According to the treatment indications and treatment uptake rates (10\% to $90 \%)$, we calculated amount of risk reduction for HCC development. When the sum of HCC risk reached 100\%, we counted one new case of HCC. For example, 100 patients with an HCC risk of 1\%

Table 2. Baseline Characteristics of the Study Cohort

\begin{tabular}{lc}
\hline \multicolumn{1}{c}{ Characteristic } & Subjects with HBsAg $(+)(\mathrm{n}=420)$ \\
\hline Male sex & $206(49)$ \\
Age, yr & $55.8 \pm 10.4$ \\
$40-49$ & $129(30.7)$ \\
$50-59$ & $148(35.2)$ \\
$\geq 60$ & $143(34.1)$ \\
AST, U/L & $29.5(27.0-31.9)$ \\
ALT, U/L & $29.7(25.4-33.9)$ \\
$<15$ & $68(16.2)$ \\
$15-44$ & $300(71.4)$ \\
$\geq 45$ & $52(12.4)$ \\
HBeAg (+)* & $66(15.7)$ \\
HBV DNA, copies/mL* & \\
$<300$ & $93(22.1)$ \\
$300-9,999$ & $125(29.8)$ \\
$10,000-99,999$ & $84(20.0)$ \\
$100,000-999,999$ & $45(10.7)$ \\
$\geq 1,000,000$ & $73(17.4)$ \\
\hline
\end{tabular}

Data are presented as number $(\%)$, mean \pm SD, or mean $(95 \%$ confidence interval).

HBsAg, hepatitis B surface antigen; AST, aspartate aminotransferase; $\mathrm{ALT}$, alanine aminotransferase; $\mathrm{HBeAg}$, hepatitis B envelope antigen; HBV, hepatitis B virus.

*HBeAg positivity and HBV DNA titer were randomly allocated based on the Risk Evaluation of Viral Load Elevation and Associated Liver Disease/Cancer-Hepatitis B Virus (REVEAL-HBV) study. ${ }^{4,7}$ corresponded to one new case of HCC within 5 years.

\section{RESULTS}

\section{Identification of candidates for antiviral therapy using the three treatment criteria}

Table 2 summarizes the baseline characteristics of the study cohort. The distribution of the laboratory results was similar to that of the REVEAL study. ${ }^{4,7}$ We investigated the proportion of potential candidates for antiviral therapy based on the three inclusion criteria. Under the Korean NHI indication, a very small proportion of subjects could be treated; only 3.76\% of the studied cohort had AST or ALT levels $>80 \mathrm{U} / \mathrm{L}$ and high serum HBV DNA levels (>20,000 IU/mL in HBeAg-positive subjects and $>2,000 \mathrm{IU} / \mathrm{mL}$ in $\mathrm{HBeAg}$-negative subjects). This corresponded to 37,321 individuals (95\% confidence interval [CI], 37,208 to $37,434)$ in the Korean HBV positive population ( $n=993,872)$. Under the EASL guidelines, 15.37\% were considered candidates for antiviral therapy. When we applied the new extended indication, the treatment candidates increased to $48.2 \%$ of the population (Table 3).

\section{The change in HCC risk before and after antiviral therapy}

We investigated the risk reduction of HCC development after antiviral treatment. If we defined the 5-year risk of HCC development of at-risk and super-high-risk patients as more than $1 \%$ and $10 \%$, respectively, about half of the subjects (47.0\%) fell into the at-risk group. The super-high-risk patients included $5.7 \%$ of all subjects. The total 5-year risk was 2.73\% $(2,725$ cases per 100,000 persons; $95 \%$ CI, 2,708 to 2,742 per 100,000).

As expected, the HCC risk was reduced remarkably after treatment. The more patients treated, the greater the reduction in HCC risk that was observed. We compared HCC risks after treatment among the three indication criteria. If HBsAg-positive Korean adults ( $>40$ years old) were treated as per the Korean NHI indication, the effect on HCC reduction was minimal. The total 5-year risk was 2.53\% (2,531 cases per 100,000 persons; $95 \%$ CI, 2,524 to 2,538 per 100,000). Only $7.1 \%$ of HCC cases were prevented, even when 100\% of the indicated patients were

Table 3. Patients Eligible for Antiviral Therapy in the General Korean Population with Chronic HBV Infection and Prediction of Risk Reduction for HCC Development after 5 Years of Antiviral Therapy

\begin{tabular}{|c|c|c|c|c|}
\hline \multirow{2}{*}{ Criteria } & \multicolumn{2}{|c|}{ Eligible patients for antiviral therapy } & \multirow{2}{*}{ HCC risk reduction, \% } & \multirow{2}{*}{ p-value* } \\
\hline & Proportion, \% & No. & & \\
\hline Korean NHI & $3.76 \pm 0.03$ & $37,321(37,208-37,434)$ & $-7.14 \pm 0.14$ & $<0.0001$ \\
\hline EASL & $15.37 \pm 0.06$ & $152,797(152,539-153,056)$ & $-23.33 \pm 0.26$ & \\
\hline New extended & $48.20 \pm 0.23$ & $479,085(478,066-480,105)$ & $-58.82 \pm 0.07$ & \\
\hline
\end{tabular}

Data are presented as mean \pm SD or mean (95\% confidence interval).

HBV, hepatitis B virus; HCC, hepatocellular carcinoma; NHI, National Health Insurance of Korea; EASL, European Association for the Study of the Liver.

*One-way ANOVA was performed. 
treated (Fig. 2). Under the EASL guidelines, the total 5-year risk was 2.09\% (2,089 cases per 100,000 persons; 95\% CI, 2,082 to $2,096$ per 100,000$)$. About one quarter of HCC cases (23.3\%) were prevented. If the extended indication was used, the total 5-year risk was $1.12 \%$ (1,122 cases per 100,000 persons; 95\% CI, 1,120 to 1,124 per 100,000 ), meaning that $58.8 \%$ of HCC cases could be prevented in 5 years by extending the treatment indications.

\section{The change in HCC risk according to treatment uptake rate}

Since $100 \%$ of indicated patients could not receive treatment, we investigated the treatment outcomes according to the treatment uptake rate. A rate of 60\% meant that 60 of 100 indicated patients were treated. The estimated HCC-risk reduction was summarized according to treatment uptake rates (10\% to 90\%)

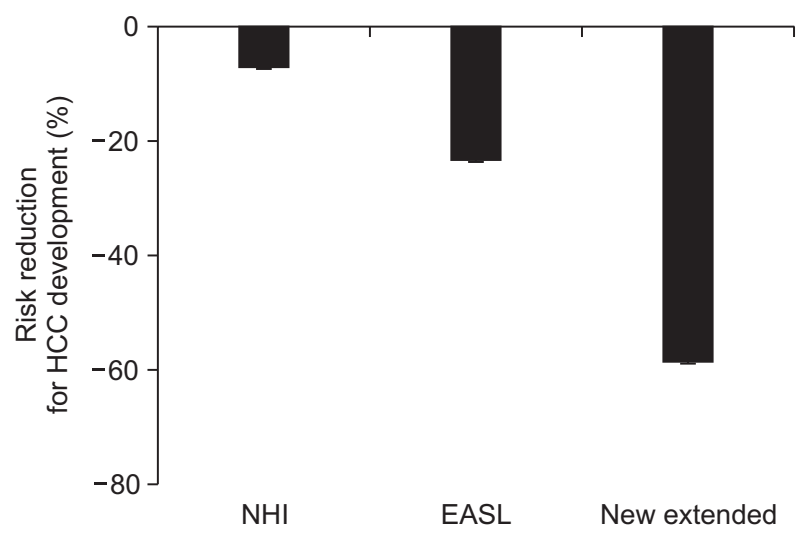

Fig. 2. The change in hepatocellular carcinoma (HCC) risk after antiviral therapy according to three treatment criteria.

NHI, National Health Insurance; EASL, European Association for the Study of the Liver. and treatment indication (Table 4). The HCC risk decreased by $0.74 \%, 2.39 \%$, and 5.87\% per 10\% increase in the treatment uptake rate under the Korean NHI, EASL, and extended criteria, respectively. This meant that 20, 65, and 160 new HCC cases could be prevented among 100,000 Korean individuals ( $>40$ years) with chronic HBV infection under the respective treatment criteria.

\section{DISCUSSION}

In this study, we estimated and compared the antiviral effects of three treatment indications in the general Korean population. As expected, the Korean NHI indication was the most stringent and resulted in $<4 \%$ of the population being candidates for antiviral therapy. Because the baseline data originated from a crosssectional survey, this percentage might increase during longterm follow up. However, on the other hand, a moderate number of patients might be lost to follow-up if not treated in the early period and only requested to attend periodic examination. The EASL guidelines are more permissive for antiviral therapy; four times more patients were eligible for antiviral therapy under the EASL criteria than under the Korean NHI indication. Thus, the number of eligible patients is very sensitive to the antiviral indications used. In particular, expanding antiviral indication is closely related with a markedly reduced risk of developing HCC. More HCC cases are prevented in the general population when more patients are treated. New extended indication was created based on the finding that HCC risk increases significantly when HBV DNA level is $>2,000 \mathrm{IU} / \mathrm{mL}^{5,23}$ Active viral replication per se is an independent risk factor for development of HCC. Moreover, ALT levels may not increase, even in patients with significant liver fibrosis. When Asian patients over 40 years of age have normal serum ALT but a high HBV DNA level, it is

Table 4. Estimated Risk Reduction of HCC Development in the 5 -Year after Antiviral Treatment in the General Korean Population

\begin{tabular}{|c|c|c|c|c|c|c|}
\hline \multirow{2}{*}{ TUR, \% } & \multicolumn{2}{|c|}{ Korean NHI } & \multicolumn{2}{|c|}{ EASL } & \multicolumn{2}{|c|}{ New extended } \\
\hline & Mean, \% & SD & Mean, \% & SD & Mean,\% & SD \\
\hline 10 & -0.74 & 0.06 & -2.39 & 0.10 & -5.88 & 0.13 \\
\hline 20 & -1.48 & 0.07 & -4.78 & 0.15 & -11.77 & 0.18 \\
\hline 30 & -2.21 & 0.10 & -7.17 & 0.15 & -17.62 & 0.22 \\
\hline 40 & -2.95 & 0.11 & -9.60 & 0.16 & -23.48 & 0.21 \\
\hline 50 & -3.67 & 0.11 & -11.97 & 0.17 & -29.38 & 0.24 \\
\hline 60 & -4.42 & 0.10 & -14.38 & 0.17 & -35.18 & 0.22 \\
\hline 70 & -5.17 & 0.09 & -16.76 & 0.16 & -41.10 & 0.23 \\
\hline 80 & -5.89 & 0.08 & -19.14 & 0.13 & -47.02 & 0.18 \\
\hline 90 & -6.63 & 0.06 & -21.52 & 0.12 & -52.86 & 0.14 \\
\hline
\end{tabular}

TUR was the proportion of patients who participated in antiviral therapy among patients eligible for the treatment. For every $100 \%$ increase in TUR, HCC risk was reduced by $-5.87 \%,-2.39 \%$, and $-0.74 \%$ under the extended indication, the EASL guideline, and the Korean NHI indication, respectively.

HCC, hepatocellular carcinoma; TUR, treatment uptake rate; NHI, National Health Insurance; EASL, European Association for the Study of the Liver. 
uncertain whether they are really in an immune tolerant state. A systematic review showed that approximately one-fifth of patients with normal ALT levels may have significant hepatic fibrosis. ${ }^{24}$ We postulate that most patients over 40-years-of-age are at risk if their serum HBV DNA level is $>2,000 \mathrm{IU} / \mathrm{mL}$. If the new extended indication is applied to the Korean population, $50 \%$ could be eligible for treatment and the preventive effect of antiviral therapy would be $-60 \%$ of HCC development. Although the preventive effect is very large, further studies are needed because this study was based on many critical assumptions and drug costs, and potential adverse events were not included. Based on these parameters, the antiviral indications in Korea for $\mathrm{CHB}$ that are more cost-effective should be developed.

It can be argued that the long-term antiviral effect of preventing HCC in relatively low-risk patients is unknown. Due to a lack of clinical data, we assumed that the preventive effect of antiviral agents would be less in higher-risk patients. We used only five parameters to predict HCC risk: age, sex, ALT, HBeAg, and HBV-DNA, in the REACH-B score. Liver fibrosis, which can be a major risk factor for HCC, was not included in the prediction model. ${ }^{25}$ Considering the marked improvement in liver fibrosis in most treated patients, wide use of antiviral agents in relatively low-risk groups would also be beneficial in preventing advanced fibrosis. ${ }^{26}$ Moreover, we assumed that the risk would not decrease during the first 2 years of treatment. Despite our strict estimate, the dramatic decrease in HCC risk in the general population suggests that extending the antiviral indications is very important in controlling the development of HCC. Above all, the major finding of this study was that current NHI indication are insufficient to reduce the risk of HCC in the Korean population. Future studies should determine the degree to which the antiviral indication should be extended.

Extending treatment could also increase the HCC surveillance rate. Absence of symptoms, low insight, and economic burden are common barriers to optimal surveillance. Although the national liver cancer surveillance program in Korea has been conducted since 2003, the surveillance rate is only 23\%, which is much lower than that for other cancers. ${ }^{27}$ Moreover, one study showed that surveillance rates tend to decrease over time. ${ }^{28}$ As patients undergoing treatment are more committed to HCC surveillance, strategies aimed at increasing treatment in more patients may help increase the surveillance rate. As the number of patients undergoing treatment increases, more HCC cases will be detected; most patients undergoing treatment are apt to receive surveillance for HCC, as compared to untreated patients, so more HCC cases will be detected at earlier stages.

In this study, we estimated the potential effects of the current Korean NHI indication by calculating the HCC risk before and after antiviral therapy using a computer-based model. This simulation approach was possible despite limited resources. If very large clinical trials cannot be conducted to determine long-term outcomes, this approach would provide an alternative method.
If more accurate Korean data and HCC prediction models were available, a more precise prediction would be possible for the Korean population.

A limitation of this study was that we did not use actual HBeAg positivity or HBV DNA viral load data from the general Korean population because KNHANES did not include these data. Instead, we used Taiwanese data, as the two populations share similar viral transmission characteristics (neonatal infection) and Asian ethnicity. However, genotype B is more prevalent in Taiwan, whereas Korean HBVs are all genotype C. Generally, genotype $\mathrm{C}$ is associated with higher risks for cirrhosis and HCC. ${ }^{29}$ The difference in viral genotypes between the two countries could be a confounding factor in this study. To ensure more reliable health policies, accurate HBV status data are critical. KNHANES should include more HBV tests to depict current HBV infection status and predict future risk. Lastly, because the KNHANES data was based on interviews and blood tests not including imaging tests, some of cirrhosis might be unrecognized and included in this study.

In conclusion, with limited resources a computer-based modeling approach to predict outcomes is feasible. Extending the antiviral indications would decrease HCC development significantly in the general Korean population. If supported by further cost-effectiveness studies, it should be considered to extend the current Korean NHI indication.

\section{CONFLICTS OF INTEREST}

No potential conflict of interest relevant to this article was reported.

\section{ACKNOWLEDGEMENTS}

The authors would like to thank Joohyun Kim, MD, Medical College of Wisconsin, Milwaukee, WI, United States, for the preparation of the manuscript.

\section{REFERENCES}

1. Song IH, Kim KS. Current status of liver diseases in Korea: hepatocellular carcinoma. Korean J Hepatol 2009;15 Suppl 6:S50-S59.

2. Kim H, Shin AR, Chung HH, et al. Recent trends in hepatitis B virus infection in the general Korean population. Korean J Intern Med 2013;28:413-419.

3. Kim KS, Kim SH, Kim IH, et al. Report of primary liver cancer randomised registration in Korea. Seoul: The Korean Liver Cancer Study Group, National Cancer Registration Center (Korea), 2010:19.

4. Chen CJ, Yang HI, Su J, et al. Risk of hepatocellular carcinoma across a biological gradient of serum hepatitis B virus DNA level. JAMA 2006;295:65-73.

5. Yang HI, Yeh SH, Chen PJ, et al. Associations between hepatitis B 
virus genotype and mutants and the risk of hepatocellular carcinoma. J Natl Cancer Inst 2008;100:1134-1143.

6. Chen JD, Yang HI, Iloeje UH, et al. Carriers of inactive hepatitis B virus are still at risk for hepatocellular carcinoma and liver-related death. Gastroenterology 2010;138:1747-1754.

7. Yang HI, Sherman M, Su J, et al. Nomograms for risk of hepatocellular carcinoma in patients with chronic hepatitis B virus infection. J Clin Oncol 2010;28:2437-2444.

8. Yang HI, Yuen MF, Chan HL, et al. Risk estimation for hepatocellular carcinoma in chronic hepatitis B (REACH-B): development and validation of a predictive score. Lancet Oncol 2011;12:568574.

9. Hosaka T, Suzuki F, Kobayashi M, et al. Long-term entecavir treatment reduces hepatocellular carcinoma incidence in patients with hepatitis B virus infection. Hepatology 2013;58:98-107.

10. Gordon SC, Lamerato LE, Rupp LB, et al. Antiviral therapy for chronic hepatitis B virus infection and development of hepatocellular carcinoma in a US population. Clin Gastroenterol Hepatol 2014;12:885-893.

11. Wu CY, Lin JT, Ho HJ, et al. Association of nucleos(t)ide analogue therapy with reduced risk of hepatocellular carcinoma in patients with chronic hepatitis B: a nationwide cohort study. Gastroenterology 2014;147:143-151.e5.

12. Wong GL, Chan HL, Chan HY, et al. Accuracy of risk scores for patients with chronic hepatitis $\mathrm{B}$ receiving entecavir treatment. Gastroenterology 2013;144:933-944.

13. Nation Health Insurance Service (NHIS). Social security system of Korea [Internet]. Wonju: NHIS; c2014 [cited 2015 Jun 5]. Available from: http://www.nhic.or.kr/static/html/wbd/g/a/wbdga0302. html.

14. Korea Centers for Disease Control and Prevention; Ministry of Health and Welfare. The fifth Korean National Health and Nutrition Examination Survey [Internet]. Osong: Korea Centers for Disease Control and Prevention; c2014 [cited 2014 Jan 6]. Available from: https://knhanes.cdc.go.kr/knhanes/index.do.

15. Lee HS, Kim JK, Cheong JY, et al. Prediction of compensated liver cirrhosis by ultrasonography and routine blood tests in patients with chronic viral hepatitis. Korean J Hepatol 2010;16:369-375.

16. European Association for the Study of the Liver. EASL clinical practice guidelines: management of chronic hepatitis B virus infection. J Hepatol 2012;57:167-185.

17. Lee JK, Shim JH, Lee HC, et al. Estimation of the healthy upper limits for serum alanine aminotransferase in Asian populations with normal liver histology. Hepatology 2010;51:1577-1583.

18. Tenney DJ, Rose RE, Baldick CJ, et al. Long-term monitoring shows hepatitis B virus resistance to entecavir in nucleoside-naïve patients is rare through 5 years of therapy. Hepatology 2009;49: 1503-1514.

19. Gordon SC, Krastev Z, Horban A, et al. Efficacy of tenofovir disoproxil fumarate at 240 weeks in patients with chronic hepatitis B with high baseline viral load. Hepatology 2013;58:505-513.

20. Ridruejo E. Treatment of chronic hepatitis B in clinical practice with entecavir or tenofovir. World J Gastroenterol 2014;20:71697180.

21. Chang TT, Lai CL, Kew Yoon S, et al. Entecavir treatment for up to 5 years in patients with hepatitis B e antigen-positive chronic hepatitis B. Hepatology 2010;51:422-430.

22. Heathcote EJ, Marcellin P, Buti M, et al. Three-year efficacy and safety of tenofovir disoproxil fumarate treatment for chronic hepatitis B. Gastroenterology 2011;140:132-143.

23. Iloeje UH, Yang HI, Su J, et al. Predicting cirrhosis risk based on the level of circulating hepatitis B viral load. Gastroenterology 2006;130:678-686.

24. Chao DT, Lim JK, Ayoub WS, Nguyen LH, Nguyen MH. Systematic review with meta-analysis: the proportion of chronic hepatitis B patients with normal alanine transaminase $\leq 40 \mathrm{IU} / \mathrm{L}$ and significant hepatic fibrosis. Aliment Pharmacol Ther 2014;39:349358.

25. Lee HW, Yoo EJ, Kim BK, et al. Prediction of development of liverrelated events by transient elastography in hepatitis B patients with complete virological response on antiviral therapy. Am J Gastroenterol 2014;109:1241-1249.

26. Marcellin P, Gane E, Buti M, et al. Regression of cirrhosis during treatment with tenofovir disoproxil fumarate for chronic hepatitis B: a 5-year open-label follow-up study. Lancet 2013;381:468-475.

27. Park B, Choi KS, Lee YY, Jun JK, Seo HG. Trends in cancer screening rates among Korean men and women: results from the Korean National Cancer Screening Survey (KNCSS), 2004-2011. Cancer Res Treat 2012;44:113-120.

28. Wong CR, Garcia RT, Trinh HN, et al. Adherence to screening for hepatocellular carcinoma among patients with cirrhosis or chronic hepatitis B in a community setting. Dig Dis Sci 2009;54:27122721.

29. Kao JH. Hepatitis B virus genotypes and hepatocellular carcinoma in Taiwan. Intervirology 2003;46:400-407. 